\title{
Fundamental Parameters of Be Stars Seen Equator-On
}

\author{
J. Zorec ${ }^{1}$, Y. Frémat ${ }^{2}$, A.M. Hubert ${ }^{2}$, M. Floquet ${ }^{2}$ \\ ${ }^{1}$ Institut d'Astrophysique de Paris, CNRS, France; ${ }^{2}$ DASGAL, \\ Observatoire de Paris-Meudon, France
}

\begin{abstract}
The geometric deformation, as well as the non-uniform surface gravity and temperature distributions induced by the fast rotation, are taken into account to determine the fundamental parameters of $10 \mathrm{Be}$ stars seen nearly equator-on. The stars occur in the first half of the main sequence evolutionary span, which suggests that their fast rotation is related to initial formation conditions, rather than to evolutionary effects.
\end{abstract}

\section{The method}

The hemisphere-averaged photospheric-related quantities are deduced from the observed $\operatorname{BCD}\left(\lambda_{1}, D_{*}\right)$ parameters, which minimize the uncertainties introduced by the circumstellar environment (Divan \& Zorec, 1982). We assumed that the stellar core is not strongly affected by rotation. As the program stars are in the solar vicinity, we used Schaller et al.'s (1992) evolutionary tracks for rotationless stars with $Z=0.02$. The observed quantities, which are affected by rotation, and the corresponding fundamental stellar parameters for the same stars at rest, derived to locate them in Schaller's et al. evolutionary tracks, are assumed to be related as:

$$
\begin{aligned}
X_{\mathrm{obs}}\left(\lambda_{1}, D_{*}\right) & =X_{o}\left(M_{o}, \tau_{o}\right) F_{\mathrm{X}}\left(M_{o}, \omega, i, \tau_{o}\right), \\
V \sin i & =V_{\mathrm{c}}\left(M_{o}, \tau_{o}\right)\left[\frac{R_{\mathrm{e}}\left(M_{o}, \omega, \tau_{o}\right)}{R_{\mathrm{c}}\left(M_{o}, \tau_{o}\right)}\right] \omega \sin i,
\end{aligned}
$$

where $X_{\text {obs }}$ stands for: $L / L_{\odot}, D_{*}$ (BCD Balmer discontinuity (BD)) and $\lambda_{1}$ (BCD mean spectral position of BD); $X_{o}$ represents the same quantities of the rotationless object; $F_{\mathrm{X}}$ are 3 functions obtained from model spectra of rotationally distorted stars which depend on the true mass of the object $M_{o} / M_{\odot}$, the ratio $\omega=\Omega / \Omega_{\mathrm{c}}\left(\Omega_{\mathrm{c}}\right.$ is the critical angular velocity), the inclination $i$ and the age $\tau_{o}$ of the rotationally unperturbed stellar core. $V_{\mathrm{c}}$ and $R_{\mathrm{c}}$ are, respectively, the critical velocity and equatorial radius; $R_{\mathrm{e}}(\omega)$ is the stellar equatorial radius.

\section{Results and discussion}

We used Chauville et al.'s (2001) $V \sin i$. HD 10516, HD 41335 and HD 200120 are interacting binaries, so their parameters are uncertain. The observed and 
Table 1. Studied Be stars and their fundamental parameters.

\begin{tabular}{|c|c|c|c|c|c|c|c|c|c|}
\hline & & ervat & & & derive & 1 parame & eters & & \\
\hline Star & $\begin{array}{c}{\left[T_{\text {eff }}\right]} \\
\text { dex }\end{array}$ & $\begin{array}{c}L / L_{\odot} \\
\operatorname{dex}\end{array}$ & $\begin{array}{c}f_{\mathrm{ph}} \\
c^{-1}\end{array}$ & $\Omega / \Omega_{c}$ & $M / M_{\odot}$ & $R_{\mathrm{e}}(\omega) / R_{\odot}$ & $i^{\circ}$ & $\begin{array}{c}f_{\text {rot }} \\
c^{-1} d^{-1}\end{array}$ & $\begin{array}{c}\tau_{o} / 10^{7} \\
\mathrm{yr}\end{array}$ \\
\hline HD 10516 & 4.49 & 4.44 & 2.12 & $0.83 \pm 0.09$ & $16.7 \pm 2.5$ & $6.2 \pm 0.4$ & $70 \pm 17$ & $1.24 \pm 0.26$ & $<0.3$ \\
\hline HD 20336 & 4.31 & 3.50 & 2.02 & 0.82 & $7.7 \pm$ & & & $1.60 \pm$ & 0.77 \\
\hline HD 22192 & 4.21 & 3.23 & 0.97 . & $0.84 \pm 0.08$ & $5.9 \pm 0.6$ & $5.7 \pm 0.4$ & $67 \pm 16$ & $1.34 \pm 0.27$ & 4.12 \\
\hline HD 35439 & 4.38 & 4.16 & 1.60 & $0.73 \pm 0.16$ & $11.2 \pm 1.9$ & $7.3 \pm 0.6$ & $65 \pm$ & $1.06 \pm 0.23$ & 0.91 \\
\hline HD 37202 & 4.31 & 3.96 & 1.31 & $0.75 \pm 0.10$ & $9.5 \pm 0.9$ & $8.4 \pm 0.5$ & $72 \pm$ & $0.91 \pm 0.15$ & 1.86 \\
\hline HD 41335 & 4.37 & 4.04 & - & $0.86 \pm 0.09$ & $10.8 \pm 1.8$ & $6.8 \pm 0.6$ & $69 \pm$ & $1.15 \pm 0.24$ & 0.95 \\
\hline HD 6 & 4.55 & 4.45 & - & $0.74 \pm 0$ & $18.7 \pm 5.7$ & $4.7 \pm 0.6$ & $65 \pm$ & $1.67 \pm 0.37$ & - \\
\hline $\mathrm{HD}$ & 4.25 & 3.68 & - & $0.83 \pm 0.08$ & $7.7 \pm 0.9$ & $7.9 \pm 0.6$ & $70 \pm$ & $0.96 \pm 0.19$ & 2.86 \\
\hline HD & 4.37 & 3.88 & 1.77 & & $10.0 \pm 1.0$ & \pm 0.4 & $68 \pm$ & $1.33 \pm 0.27$ & 0.57 \\
\hline HD 200120 & 4.41 & 3.93 & 3.57 & $0.80 \pm 0.13$ & $11.2 \pm 2.8$ & $5.0 \pm 0.6$ & $69 \pm 21$ & $1.57 \pm 0.34$ & $<0.5$ \\
\hline
\end{tabular}

derived parameters of the stars are presented in Table1. We see that: 1) On average $\bar{\omega}=0.8 \pm 0.1$, in agreement with Chauville et al. (2001), and $\bar{i}=68^{\circ} \pm 18^{\circ}$ even for the strong Be-shell stars; 2) Most stars lie in the first half of the main sequence evolutionary span, so that they have hardly had time to become fast rotators by evolutionary effects, implying that they formed as fast rotators. Rotational frequencies are $f_{\text {rot }}=0.02 \overline{(V \sin i \pm \delta) / \sin \left(i \pm \delta_{i}\right) /\left(R_{\mathrm{e}} \pm \delta_{r}\right)} \mathrm{cd}^{-1}$. Frequencies $f_{\mathrm{ph}}$ related to periodic photometric variations (see e.g. Hubert \& Floquet, 1998) are on average $\overline{f_{\mathrm{ph}} / f_{\text {rot }}}=1.5 \pm 0.5 \mathrm{~cd}^{-1}$. If $f_{\mathrm{ph}}=f_{\text {rot }}$ is true, then either: a) $R_{\mathrm{e}}$ are correct and inclinations become $\vec{i} \simeq 38^{\circ}$, so that our Be-shell stars will rather be seen pole-on; or: b) $i$ are correct and the radii reduce to $\overline{R_{\mathrm{e}}} \simeq 0.7 \overline{R_{\mathrm{e}} \text { (used) }}$, so that stars will shrink under their ZAMS size. Moreover, if: c) stars are critical rotators and the mean inclination will be $\bar{i} \simeq$

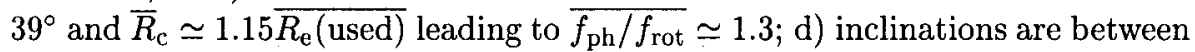
$i_{\mathrm{c}}=i(\omega=1)$ and $i=\pi / 2$, and $\overline{R_{\mathrm{e}}}=\overline{\left[R_{\mathrm{e}}(\omega=0), R_{\mathrm{e}}(\omega=1)\right]} \simeq 1.18 \overline{R_{\mathrm{e}} \text { (used) }}$, which will produce $\overline{f_{\mathrm{ph}} / f_{\text {rot }}} \simeq 1$. This means that we can hardly think of $f_{\mathrm{ph}}$ representing $f_{\text {rot }}$.

\section{References}

Chauville, J., Zorec, J., Ballereau, D., et al. 2001, A\&A, submitted Divan, L. \& Zorec, J. 1982, ESA SP-177, 101

Hubert, A.M. \& Floquet, M. 1998, A\&A, 335, 565

Schaller, G., Schaerer, D., Meynet, G., et al. 1992, A\&AS, 96, 269 